\title{
ON BALANCEDNESS AND D-COMPLETENESS OF THE SPACE OF SEMI-LIPSCHITZ FUNCTIONS
}

\author{
S. ROMAGUERA ${ }^{1}$, J. M. SÁNCHEZ-ÁLVAREZ ${ }^{1}$ and M. SANCHIS ${ }^{2}$ * \\ ${ }^{1}$ E.T.S. Ingenieros de Caminos, Departamento de Matemática Aplicada, \\ IUMPA-UPV, Universidad Politécnica de Valencia, 46071 Valencia, Spain \\ e-mail: sromague@mat.upv.es, jossnclv@mat.upv.es \\ 2 Departamento de Matemáticas, Universidad Jaume I, Campus de Riu Sec, \\ 12071 Castellón, Spain \\ e-mail: sanchis@mat.uji.es
}

(Received August 13, 2007; revised September 10, 2007; accepted September 11, 2007)

\begin{abstract}
Let $(X, d)$ be a quasi-metric space and $(Y, q)$ be a quasi-normed linear space. We show that the normed cone of semi-Lipschitz functions from $(X, d)$ to $(Y, q)$ that vanish at a point $x_{0} \in X$, is balanced. Moreover, it is complete in the sense of $\mathrm{D}$. Doitchinov whenever $(Y, q)$ is a biBanach space.
\end{abstract}

\section{Introduction}

In the last years the study of real-valued semi-Lipschitz functions defined on a $T_{0}$ quasi-pseudo-metric space has received a certain attention $[11,12,16,18]$. In particular, it was shown in [16] that the set of realvalued semi-Lipschitz functions defined on a $T_{0}$ quasi-pseudo-metric space $(X, d)$ that vanish at a point $x_{0} \in X$ can be structured as a normed cone. Applications of semi-Lipschitz functions to questions on best approximation, global attractors on dynamical systems, and concentration of measure can be found in [13, 16], [17] and [22], respectively.

*The authors acknowledge the support of Plan Nacional $\mathrm{I}+\mathrm{D}+\mathrm{I}$ and FEDER, under grant MTM2006-14925-C02-01. The second listed author is also supported by a grant FPI from the Spanish Ministry of Education and Science.

Key words and phrases: semi-Lipschitz function, quasi-normed linear space, quasi-metric, balanced, biBanach, D-complete.

2000 Mathematics Subject Classification:54E50, 54C35, 46B20, 46E15.

0236-5294/\$20.00 (C) 2008 Akadémiai Kiadó, Budapest 
In [21], semi-Lipschitz functions that are valued in a quasi-normed linear space have been discussed. This study was motivated, in great part, by the fact that quasi-normed linear spaces provide suitable mathematical models in the theory of computational complexity (see [4, 5, 20]).

Here we obtain some new properties of the space $S L_{0}(d, q)$ of semiLipschitz functions defined on the quasi-metric space $(X, d)$ with values in the quasi-normed linear space $(Y, q)$ and that vanish at a point $x_{0} \in X$. We show the somewhat surprising fact that $S L_{0}(d, q)$ is balanced in the sense of Doitchinov [2]. We also prove that it is complete in the sense of Doitchinov whenever $(Y, q)$ is a biBanach space. As an application of these results to asymmetric functional analysis, we deduce that the dual space of a $T_{1}$ quasi-normed linear space is balanced and Doitchinov complete. It is interesting to recall that the study of balanced quasi-metric spaces from a fuzzy point of view has been recently started in $[8,19]$, and that, on the other hand, some applications of balanced (extended) quasi-metrics to theoretical computer science have been given in [14, 15].

Throughout this paper the letters $\mathbb{R}^{+}$and $\mathbb{N}$ will denote the set of nonnegative real numbers and the set of positive integers numbers, respectively. Our basic reference for quasi-metric spaces is [3].

Next we recall some pertinent concepts.

As usual by a monoid we mean a semigroup $(X,+)$ with neutral element.

According to [9] a cone (semilinear space in [16]) is a triple $(X,+, \cdot)$ such that $(X,+)$ is an Abelian monoid, and - is a function from $\mathbb{R}^{+} \times X$ to $X$ such that for all $x, y \in X$ and $r, s \in \mathbb{R}^{+}:$(i) $r \cdot(s \cdot x)=(r s) \cdot x$; (ii) $r \cdot(x+y)$ $=(r \cdot x)+(r \cdot y)$; (iii) $(r+s) \cdot x=(r \cdot x)+(s \cdot x)$; (iv) $1 \cdot x=x$.

A quasi-norm on a cone $(X,+, \cdot)$ is $[16,18]$ a function $q: X \rightarrow \mathbb{R}^{+}$such that for all $x, y \in X$ and $r \in \mathbb{R}^{+}:$(i) $x=\mathbf{0}$ if and only if there is $-x \in X$ and $q(x)=q(-x)=0$; (ii) $q(r \cdot x)=r q(x)$; (iii) $q(x+y) \leqq q(x)+q(y)$.

If the quasi-norm $q$ satisfies: ( $\left.\mathrm{i}^{\prime}\right) q(x)=0$ if and only if $x=\mathbf{0}$, then $q$ is called a norm on the cone $(X,+, \cdot)$.

A (quasi-)normed cone is a pair $(X, q)$ such that $X$ is a cone and $q$ is a (quasi-)norm on $X$.

If $(X,+, \cdot)$ is a linear space and $q$ is a quasi-norm on $X$, then the pair $(X, q)$ is called a quasi-normed linear space (asymmetric normed linear space in [4]). Note that, in this case, the function $q^{-1}: X \rightarrow \mathbb{R}^{+}$given by $q^{-1}(x)$ $=q(-x)$ is also a quasi-norm on $X$ and the function $q^{s}: X \rightarrow \mathbb{R}^{+}$given by $q^{s}(x)=\max \{q(x), q(-x)\}$ is a norm on $X$. As in [6], we say that $(X, q)$ is a biBanach space if $\left(X, q^{s}\right)$ is a Banach space.

An easy but crucial example of a biBanach space is the pair $(\mathbb{R}, u)$, where $u$ is the quasi-norm on $\mathbb{R}$ given by $u(x)=\max \{x, 0\}$ for all $x \in \mathbb{R}$. Note that $u^{s}(x)=|x|$ for all $x \in \mathbb{R}$, so $(\mathbb{R}, u)$ is a biBanach space. 
Let us recall that a quasi-pseudo-metric on a set $X$ is a function $d$ : $X \times X \rightarrow \mathbb{R}^{+}$such that for all $x, y, z \in X$ : (i) $d(x, x)=0$; (ii) $d(x, z) \leqq d(x, y)$ $+d(y, z)$. If $d$ satisfies the additional condition: (iii) $d(x, y)=0$ if and only if $x=y$, then we will say that $d$ is a quasi-metric on $X$.

We will also consider extended quasi-(pseudo-)metrics. They satisfy the above three axioms, except that we allow $d(x, y)=+\infty$.

If $d$ is a(n extended) quasi-(pseudo-)metric, then the function $d^{-1}$ defined on $X \times X$ by $d^{-1}(x, y)=d(y, x)$ is also a(n extended) quasi-(pseudo-) metric called the conjugate of $d$ and $d^{s}$ defined on $X \times X$ by $d^{s}(x, y)$ $=\max \{d(x, y), d(y, x)\}$, is a(n extended) (pseudo-)metric on $X$.

$\mathrm{A}(\mathrm{n}$ extended) quasi-(pseudo-)metric space is a pair $(X, d)$ such that $X$ is a set and $d$ is a(n extended) quasi-(pseudo-)metric on $X$.

Each (extended) quasi-pseudo-metric $d$ on a set $X$ generates a topology $\tau(d)$ on $X$ which has as a base the family of open $d$-balls $\left\{B_{d}(x, r): x \in X\right.$, $r>0\}$, where $B_{d}(x, r)=\{y \in X: d(x, y)<r\}$ for all $x \in X$ and $r>0$. If the topology $\tau(d)$ is $T_{0}$ we say that $(X, d)$ is a $T_{0}$ (extended) quasi-pseudometric space. Observe that if $d$ is a(n extended) quasi-metric, then $\tau(d)$ is a $T_{1}$ topology on $X$.

It is well known that each quasi-norm $q$ on a linear space $X$ induces a $T_{0}$ quasi-pseudo-metric $d_{q}$ on $X$ given by $d_{q}(x, y)=q(x-y)$ for all $x, y \in X$.

\section{The results}

Let $(X, d)$ be a quasi-metric space and let $(Y, q)$ be a quasi-normed linear space. A function $f: X \rightarrow Y$ is called semi-Lipschitz if there is $k \geqq 0$ such that $q(f(x)-f(y)) \leqq k d(x, y)$ for all $x, y \in X$.

Given the quasi-metric space $(X, d)$ and the quasi-normed linear space $(Y, q)$, fix $x_{0} \in X$ and put

$$
S L_{0}(d, q)=\left\{f: X \rightarrow Y: f\left(x_{0}\right)=0 \quad \text { and } \quad \sup _{x \neq y} \frac{q(f(x)-f(y))}{d(x, y)}<\infty\right\} .
$$

Clearly $S L_{0}(d, q)$ is exactly the set of all semi-Lipschitz functions from $(X, d)$ to $(Y, q)$ that vanishes at $x_{0}$, and $\left(S L_{0}(d, q),+, \cdot\right)$ is a cone, where for each $f, g \in S L_{0}(d, q)$ and $r \in \mathbb{R}^{+}$we define $f+g$ and $r \cdot f$ in the usual pointwise way [21].

Observe that the definition of $S L_{0}(d, q)$ given here is slightly different from the ones given in [18]. This is due to the fact that quasi-metric spaces of [18] correspond to our $T_{0}$ quasi-pseudo-metric spaces. 
Now for each $f, g \in S L_{0}(d, q)$ define

$$
\rho_{d, q}(f, g)=\sup _{x \neq y} \frac{q((f-g)(x)-(f-g)(y))}{d(x, y)} .
$$

Then $\rho_{d, q}$ is an extended quasi-metric on $S L_{0}(d, q)$ and the function $\|\cdot\|_{d, q}: S L_{0}(d, q) \rightarrow \mathbb{R}^{+}$given by $\|f\|_{d, q}=\rho_{d, q}(f, \mathbf{0})$, for all $f \in S L_{0}(d, q)$ is a norm on the cone $S L_{0}(d, q)$, (compare [16, 18, 21]).

In [2] Doitchinov introduced an important property of symmetry in quasimetric spaces, namely balancedness, to develop a satisfactory theory of completion. He observed that paradigmatic examples of quasi-metric spaces, like the Sorgenfrey line, the Kofner plane and the Pixley-Roy spaces are balanced, and proved that every balanced quasi-metric generates a Hausdorff and completely regular topology.

Recall that an extended quasi-metric space $(X, d)$ is balanced provided that for each pair of sequences $\left(y_{n}\right)_{n},\left(x_{n}\right)_{n}$ in $X$ such that $\lim _{n, m \rightarrow \infty} d\left(y_{m}, x_{n}\right)$ $=0$, and each $x, y \in X$ and $r_{1}, r_{2} \in \mathbb{R}^{+}$satisfying $d\left(x, x_{n}\right) \leqq r_{1}$ and $d\left(y_{n}, y\right)$ $\leqq r_{2}$ for all $n \in \mathbb{N}$, it follows that $d(x, y) \leqq r_{1}+r_{2}$. In this case, $d$ is called a balanced extended quasi-metric.

We say that the normed cone $\left(S L_{0}(d, q),\|\cdot\|_{d, q}\right)$ is balanced if the extended quasi-metric $\rho_{d, q}$ is balanced on $S L_{0}(d, q)$.

According to [2], by a Cauchy sequence in an extended quasi-metric space $(X, d)$ we mean a sequence $\left(x_{n}\right)_{n}$ in $X$ for which there is a sequence $\left(y_{n}\right)_{n}$ in $X$ satisfying $\lim _{n, m \rightarrow \infty} d\left(y_{m}, x_{n}\right)=0$. The extended quasi-metric space $(X, d)$ is said to be complete if every Cauchy sequence is convergent with respect to $\tau(d)$.

Then, Doitchinov proved that each balanced quasi-metric space $(X, d)$ is isometrically isomorphic to a $\tau(d)$ and $\tau\left(d^{-1}\right)$-dense subspace of a balanced complete quasi-metric space.

Following the modern terminology [10], Cauchy sequences in the sense of Doitchinov will be called, in the sequel, D-Cauchy sequences and complete extended quasi-metric spaces will be called $D$-(sequentially) complete extended quasi-metric spaces. We say that the normed cone $\left(S L_{0}(d, q),\|\cdot\|_{d, q}\right)$ is Dcomplete if the extended quasi-metric $\rho_{d, q}$ is D-complete.

TheOrem 1. Let $(X, d)$ be a quasi-metric space, $(Y, q)$ a quasi-normed linear space and $x_{0} \in X$. Then $\left(S L_{0}(d, q),\|\cdot\|_{d, q}\right)$ is a balanced normed cone.

ProOF. Let $\left(f_{n}\right)_{n},\left(g_{n}\right)_{n}$ be sequences in $S L_{0}(d, q)$ with

$$
\lim _{n, m \rightarrow \infty} \rho_{d, q}\left(g_{m}, f_{n}\right)=0
$$


and let $f, g \in S L_{0}(d, q)$ and $r_{1}, r_{2} \in \mathbb{R}^{+}$such that $\rho_{d, q}\left(f, f_{n}\right) \leqq r_{1}$ and $\rho_{d, q}\left(g_{n}, g\right) \leqq r_{2}$ for all $n \in \mathbb{N}$. Choose $x, y \in X$ with $x \neq y$. Then

$$
q\left(\left(f-f_{n}\right)(x)-\left(f-f_{n}\right)(y)\right) \leqq r_{1} d(x, y),
$$

and

$$
q\left(\left(g_{n}-g\right)(x)-\left(g_{n}-g\right)(y)\right) \leqq r_{2} d(x, y),
$$

for all $n \in \mathbb{N}$. Moreover, for an arbitrary $\varepsilon>0$ there is $n_{0} \in \mathbb{N}$ such that

$$
q\left(\left(g_{n}-f_{n}\right)(y)-\left(g_{n}-f_{n}\right)(x)\right)<\varepsilon d(y, x),
$$

for all $n \geqq n_{0}$. Consequently

$$
\begin{gathered}
q((f-g)(x)-(f-g)(y)) \leqq\left\{q\left(\left(f-f_{n_{0}}\right)(x)-\left(f-f_{n_{0}}\right)(y)\right)\right. \\
\left.+q\left(\left(f_{n_{0}}-g_{n_{0}}\right)(x)-\left(f_{n_{0}}-g_{n_{0}}\right)(y)\right)+q\left(\left(g_{n_{0}}-g\right)(x)-\left(g_{n_{0}}-g\right)(y)\right)\right\} \\
<r_{1} d(x, y)+\varepsilon d(y, x)+r_{2} d(x, y) .
\end{gathered}
$$

Since $\varepsilon$ is arbitrary, it follows that

$$
q((f-g)(x)-(f-g)(y)) \leqq r_{1} d(x, y)+r_{2} d(x, y) .
$$

Therefore $\rho_{d, q}(f, g) \leqq r_{1}+r_{2}$. We conclude that $\left(S L_{0}(d, q),\|\cdot\|_{d, q}\right)$ is balanced.

Corollary. Let $(X, d)$ be a quasi-metric space, $(Y, q)$ a quasi-normed linear space and $x_{0} \in X$. Then $\left(S L_{0}(d, q), \tau\left(\rho_{d, q}\right)\right)$ is a Hausdorff and completely regular topological space.

Theorem 2. Let $(X, d)$ be a quasi-metric space, $(Y, q)$ a biBanach space and $x_{0} \in X$. Then $\left(S L_{0}(d, q),\|\cdot\|_{d, q}\right)$ is D-complete.

Proof. Let $\left(f_{n}\right)_{n}$ be a D-Cauchy sequence in $S L_{0}(d, q)$. Then, there is a sequence $\left(g_{n}\right)_{n}$ in $S L_{0}(d, q)$ such that $\lim _{n, m \rightarrow \infty} \rho_{d, q}\left(g_{m}, f_{n}\right)=0$. Thus, given $\varepsilon>0$ there is $n_{0} \in \mathbb{N}$ such that $\rho_{d, q}\left(g_{m}, f_{n}\right)<\varepsilon$ for all $n, m \geqq n_{0}$.

Now fix $x \in X$. Then

$$
q\left(\left(g_{m}-f_{n}\right)(x)\right)<\varepsilon d\left(x, x_{0}\right) \text { and } q\left(\left(f_{n}-g_{m}\right)(x)\right)<\varepsilon d\left(x_{0}, x\right),
$$

so,

$$
q^{s}\left(\left(g_{m}-f_{n}\right)(x)\right)<\varepsilon d^{s}\left(x, x_{0}\right) \text { for all } n, m \geqq n_{0} .
$$


Therefore, for each $n, m \geqq n_{0}$,

$$
q^{s}\left(\left(f_{n}-f_{m}\right)(x)\right) \leqq q^{s}\left(\left(f_{n}-g_{n_{0}}\right)(x)\right)+q^{s}\left(\left(g_{n_{0}}-f_{m}\right)(x)\right)<2 \varepsilon d^{s}\left(x, x_{0}\right),
$$

and, since $(Y, q)$ is a biBanach space, the sequence $\left(f_{n}(x)\right)_{n}$ is convergent in $\left(Y, q^{s}\right)$. Then, we can construct a function $f: X \rightarrow Y$ such that $\left(f_{n}\right)_{n}$ is pointwise convergent to $f$ with respect to the norm $q^{s}$. Observe that, by condition $(*)$, the sequence $\left(g_{n}\right)_{n}$ is also pointwise convergent to $f$ with respect to $q^{s}$.

We shall prove that $f \in S L_{0}(d)$ and that $\lim _{n \rightarrow \infty} \rho_{d, q}\left(f, f_{n}\right)=0$. Indeed, first note that $f\left(x_{0}\right)=\mathbf{0}$ because $f_{n}\left(x_{0}\right)=\mathbf{0}$ for all $n \in \mathbb{N}$. Now, for the given $\varepsilon>0$, for $n \geqq n_{0}$ and for $x, y \in X$ with $x \neq y$, there exists $m \geqq n$ such that

$$
q^{s}\left(\left(f-g_{m}\right)(x)\right)<\varepsilon d(x, y) \quad \text { and } \quad q^{s}\left(\left(f-g_{m}\right)(y)\right)<\varepsilon d(x, y) .
$$

Hence

$$
\begin{gathered}
\frac{q\left(\left(f-f_{n}\right)(x)-\left(f-f_{n}\right)(y)\right)}{d(x, y)} \\
\leqq \frac{q\left(\left(f-g_{m}\right)(x)-\left(f-g_{m}\right)(y)\right)}{d(x, y)}+\frac{q\left(\left(g_{m}-f_{n}\right)(x)-\left(g_{m}-f_{n}\right)(y)\right)}{d(x, y)} \\
<\frac{q^{s}\left(\left(f-g_{m}\right)(x)\right)+q^{s}\left(\left(f-g_{m}\right)(y)\right)}{d(x, y)}+\varepsilon<3 \varepsilon .
\end{gathered}
$$

It then follows that

$$
\sup _{x \neq y} \frac{q(f(x)-f(y))}{d(x, y)} \leqq 3 \varepsilon+\sup _{x \neq y} \frac{q\left(f_{n_{0}}(x)-f_{n_{0}}(y)\right)}{d(x, y)} .
$$

Thus, we have shown that $f \in S L_{0}(d, q)$ and $\rho_{d, q}\left(f, f_{n}\right) \leqq 3 \varepsilon$ for all $n \geqq n_{0}$. Consequently $\left(S L_{0}(d, q),\|\cdot\|_{d, q}\right)$ is D-complete.

As an application of the above results we next show that if $(X, p)$ is a $T_{1}$ quasi-normed linear space (i.e. the quasi-pseudo-metric $d_{p}$ induced by the quasi-norm $p$ is actually a quasi-metric), then the dual space $\left(X^{*}, p^{*}\right)$ of $(X, p)$ is balanced and D-complete in the natural sense that we explain in the following.

Let us recall $[1,6]$ that if $(X, p)$ is a quasi-normed linear space then the socalled dual algebraic of $(X, p)$ is the cone $X^{*}$ consisting of all linear real-valued functions on $X$ that are upper semicontinuous on $\left(X, \tau\left(\left(d_{p}\right)^{-1}\right)\right)$. Equivalently, $X^{*}$ consists of all linear real-valued functions on $X$ that are lower 
semicontinuous on $\left(X, \tau\left(d_{p}\right)\right)[18$, p. 58]. It immediately follows $[13,18]$ that $X^{*}=L(X) \cap S L_{0}\left(d_{p}, u\right)$, where $L(X)$ denotes the space of all linear realvalued functions on $X$ and $S L_{0}\left(d_{p}, u\right)$ denotes the space of all semi-Lipschitz functions from $\left(X, d_{p}\right)$ to the biBanach space $(\mathbb{R}, u)$ (see Section 1$)$ that vanish at $\mathbf{0}$. Note that in this case we have

$$
\rho_{d_{p}, u}(f, g)=\sup _{x \neq y} \frac{((f-g)(x)-(f-g)(y)) \vee 0}{p(x-y)},
$$

for all $f, g \in S L_{0}\left(d_{p}, u\right)$.

Let us also recall that $p^{*}$ is the function from $X^{*}$ to $\mathbb{R}^{+}$defined by $p^{*}(f)$ $=\sup \{f(x): p(x) \leqq 1\}$ for all $f \in X^{*}[1,6]$, and thus $\left(X^{*}, p^{*}\right)$ is a normed cone which is said to be the dual space of $(X, p)$. Furthermore $p *(f)=\|f\|_{d, u}$ for all $f \in X^{*}\left[18\right.$, p. 58], and clearly, $d_{p^{*}}(f, g)=\rho_{d_{p}, u}(f, g)$ for all $f, g \in X^{*}$, where, as in the case of quasi-normed linear spaces, we define $d_{p *}(f, g)=$ $\sup \{(f-g)(x): p(x) \leqq 1\}$.

Theorem 3. Let $(X, p)$ be a $T_{1}$ quasi-normed linear space. Then $\left(X^{*}, d_{p^{*}}\right)$ is a balanced D-complete extended quasi-metric space.

Proof. By Theorems 1 and 2, $\left(S L_{0}\left(d_{p}, u\right), \rho_{d_{p}, u}\right)$ is a balanced Dcomplete extended quasi-metric space. Since balancedness is a hereditary property, then $\left(X^{*}, d_{p^{*}}\right)$ is balanced. It remains to show that $\left(X^{*}, d_{p^{*}}\right)$ is D-complete. To this end, let $\left(f_{n}\right)_{n}$ be a D-Cauchy sequence in $\left(X^{*}, d_{p *}\right)$. Then $\left(f_{n}\right)_{n}$ is a D-Cauchy sequence in $\left(S L_{0}\left(d_{p}, u\right), \rho_{d, u}\right)$, so there is $f \in S L_{0}\left(d_{p}, u\right)$ such that $\lim _{n \rightarrow \infty} \rho_{d, u}\left(f, f_{n}\right)=0$. Moreover, and following the proof of Theorem 2, the sequence $\left(f_{n}(x)\right)_{n}$ is pointwise convergent to $f(x)$ with respect to the Euclidean norm $u^{s}$, for all $x \in X$. Taking into account this fact, it is routine to see that $f$ is a linear function. We conclude that $f \in X^{*}$ and thus $\left(X^{*}, d_{p^{*}}\right)$ is D-complete.

In the light of Theorem 3 it seems interesting to recall that there exist $T_{1}$ (actually Hausdorff) quasi-normed nonnormable linear spaces in abundance (see, for instance, [7]).

The authors are grateful to the referee for comments and suggestions which permitted them to improve the first version of the paper.

\section{References}

[1] C. Alegre, J. Ferrer and V. Gregori, On the Hahn-Banach theorem in certain linear quasi-uniform structures, Acta Math. Hungar., 82 (1999), 325-330.

[2] D. Doitchinov, On completeness in quasi-metric spaces, Topology Appl., 30 (1988), $127-148$. 
[3] P. Fletcher and W. F. Lindgren, Quasi-Uniform Spaces, Marcel Dekker (New York, 1982).

[4] L. M. García-Raffi, S. Romaguera and E. A. Sánchez-Pérez, Sequence spaces and asymmetric norms in the theory of computational complexity, Math. Comput. Model., 36 (2002), 1-11.

[5] L. M. García-Raffi, S. Romaguera and E. A. Sánchez-Pérez, The supremum asymmetric norm on sequence algebras: a general framework to measure complexity distances, Electronic Notes in Theoretical Computer Science, 74 (2003), 39-50.

[6] L. M. García-Raffi, S. Romaguera and E. A. Sánchez-Pérez, The dual space of an asymmetric normed linear space, Quaestiones Math., 26 (2003), 83-96.

[7] L. M. García-Raffi, S. Romaguera and E. A. Sánchez-Pérez, On Hausdorff asymmetric normed linear spaces, Houston J. Math., 29 (2003), 717-728.

[8] V. Gregori, J. A. Mascarell and A. Sapena, On completion of fuzzy quasi-metric spaces, Topology Appl., 153 (2005), 886-899.

[9] K. Keimel and W. Roth, Ordered Cones and Approximation, Lecture Notes Mathematics, vol. 1517, Springer (Berlin, 1992).

[10] H. P. A. Künzi, Nonsymmetric distances and their associated topologies: About the origins of basic ideas in the area of asymmetric topology, in: Handbook of the History of General Topology (eds. C. E. Aull and R. Lowen), vol. 3, Hist. Topol. 3, Kluwer Acad. Publ. (Dordrecht, 2001), pp. 853-968.

[11] C. Mustăta, Extensions of semi-Lipschitz functions on quasi-metric spaces, $A n n . \mathrm{Nu}$ mer. Theory Approx., 30 (2001), 61-67.

[12] C. Mustăta, On the extremal semi-Lipschitz functions, Ann. Numer. Theory Approx., 31 (2002), 103-108.

[13] C. Mustăta, A Phelps type theorem for spaces with asymmetric norm, Bul. Ştiinţ. Univ. Baia Mare, Ser. B, Matematică-Informatică, 18 (2002), 275-280.

[14] J. Rodríguez-López, S. Romaguera and O. Valero, Denotational semantics for programming languages, balanced quasi-metrics and fixed points, Internat. J. Comput. Math., to appear.

[15] S. Romaguera, E. A. Sánchez-Pérez and O. Valero, Computing complexity distances between algorithms, Kybernetika, 36 (2003), 569-582.

[16] S. Romaguera and M. Sanchis, Semi-Lipschitz functions and best approximation in quasi-metric spaces, J. Approx. Theory, 103 (2000), 292-301.

[17] S. Romaguera and M. Sanchis, Applications of utility functions defined on quasi-metric spaces, J. Math. Anal. Appl., 283 (2003), 219-235.

[18] S. Romaguera and M. Sanchis, Properties of the normed cone of semi-Lipschitz functions, Acta Math. Hungar., 108 (2005), 55-70.

[19] S. Romaguera, A. Sapena and O. Valero, Quasi-uniform isomorphisms in fuzzy quasi-metric spaces, bicompletion and D-completion, Acta Math. Hungar., 114 (2007), 49-60.

[20] S. Romaguera and M. Schellekens, Duality and quasi-normability for complexity spaces, Appl. Gen. Topology, 3 (2002), 91-112.

[21] J. M. Sánchez-Âlvarez, On semi-Lipschitz functions with values in a quasi-normed linear space, Appl. Gen. Topology, 6 (2005), 217-228.

[22] A. Stojmirovič, Quasi-metric space with measure, Topology Proc., 28 (2004), 655-671. 